\title{
Recurrence of Cortical Venous Haemangioma in the Tibia: Interest of Sclerotherapy
}

\author{
Mohamed Bouabdellah1, Madiha Mahfoudhi2*, Imen Gorsane², Hatem Rajhi' ${ }^{3}$, \\ Wael Chabbi', Ramzi Bouzidi' \\ ${ }^{1}$ Orthopedy Department, Charles Nicolle Hospital, Tunis, Tunisia \\ ${ }^{2}$ Internal Medicine A Department, Charles Nicolle Hospital, Tunis, Tunisia \\ ${ }^{3}$ Radiology Department, Charles Nicolle Hospital, Tunis, Tunisia \\ Email: ${ }^{*}$ madiha mahfoudhi@yahoo.fr
}

Received 19 May 2015; accepted 14 September 2015; published 17 September 2015

Copyright (C) 2015 by authors and Scientific Research Publishing Inc.

This work is licensed under the Creative Commons Attribution International License (CC BY).

http://creativecommons.org/licenses/by/4.0/

(c) (i) Open Access

\begin{abstract}
We report on a rare case of an intra-osseous haemangioma involving the medial portion of tibia in a 29-year-old man. The aim is to emphasize on diagnosis difficulties, possible recurrence and interest of sclerotherapy. In radiographs, the lesion was a well-defined osteolytic lesion having 3 mm of diameter with perimeter's sclerosis. The computed tomography images demonstrated an intra-medullar gap's image of the tibia with cortical participation. An osteoid osteoma was evocated but eliminated by the histhological examination. The osseous scintigraphy didn't find any anomaly. The outcome was marked by the recurrence of pain. T1-weighted Magnetic resonance imaging showed a hypointense lesion, while T2-weighted images revealed hyperintense areas, with internal, hypointense septa. Gadolinium-enhanced T1-weighted images showed lattice-like enhancement of the lesion. These images signed a recurrence of the intra-osseous venous haemangioma. The sclerotherapy improved the pain in three days and prevented another recurrence.
\end{abstract}

\section{Keywords}

Intra-Osseous Haemangioma, Computed Tomography, Magnetic Resonance Imaging, Tibia, Sclerotherapy

\section{Introduction}

The majority of haemangiomas that involve bone are discovered incidentally in asymptomatic patients [1]-[4].

"Corresponding author.

How to cite this paper: Bouabdellah, M., Mahfoudhi, M., Gorsane, I., Rajhi, H., Chabbi, W. and Bouzidi, R. (2015) Recurrence of Cortical Venous Haemangioma in the Tibia: Interest of Sclerotherapy. International Journal of Clinical Medicine, 6, 661666. http://dx.doi.org/10.4236/ijcm.2015.69088 
Men are affected twice as often as women, and lesions are usually discovered in the 4th-5th decades of life. Soft-tissue components may also be associated with these lesions. Osseous haemangioma is particularly common in the spine and calvaria and less frequently affects long bones such as the tibia, femur, and humerus [1][4].

\section{Case Report}

A 29-year-old patient, with no particular history, complained of pain in the right leg 20 days ago with a nocturnal recrudescence, with neither fever nor trauma. The clinical examination revealed a good general condition, a painful centimetric antero-internal tumefaction of the right leg, having an osseous consistency without inflammatory signs.

Conventional radiography of the leg showed an intracortical hair image of medial cortical with peripheral sclerosis (Figure 1). A computed tomographic scan revealed a radiolucent intramedullary gap's image of the antero-internal cortex with longitudinal spotty internal calcification (Figure 2(a), Figure 2(b)). The osseous scintigraphy was without hyper-fixation anomaly. A clinical diagnosis of atypical intramedullary osteoid osteoma was retained. The lesion was completely excised for histological examination. No reconstruction was necessary after the excision. A pathological diagnosis of intramedullary veinous haemangioma was retained. The outcome was marked four months after by recurrence of leg pain. The radiography and the computed tomography scan found a recurrence of the lesion. T1-weighted Magnetic resonance imaging showed a hypo intense lesion, while T2-weighted images revealed hyper intense areas, with an internal hypointense lesion. Gadolinium-enhanced T1-weighted images showed a lattice-like enhancement of the lesion (Figure 3(a), Figure 3(b)). These images proved the recurrence of the intra-osseous venous haemangioma; Technique: A sclerotherapy guided by the computed tomography scan under local anesthesia, scannographic identification of the lesion was carried out bya transcortical pin (Figure 4(a)). The journey was enlarged by a strawberry (Figure 4(b)). A catheterization of the angioma was performed by a probe guided by the pin (Figure 4(c)).

Sclerotherapy under pressure with scannographic control was finally performed. This technique improved the osseous pain which disappeared within 72 hours. The 18-month follow-up was characterised by the absence of pain and the professional activities were resumed.

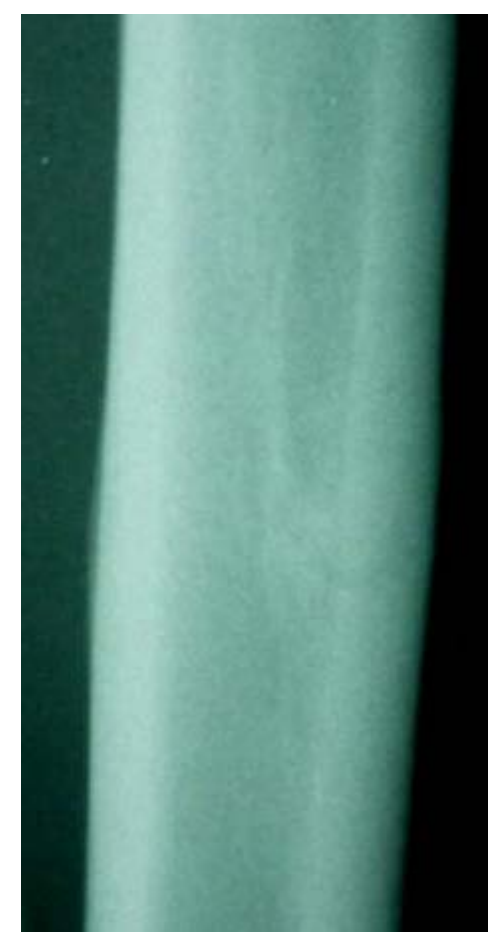

Figure 1. Intracortical medial crack lesion of leg with characteristic condensation. 


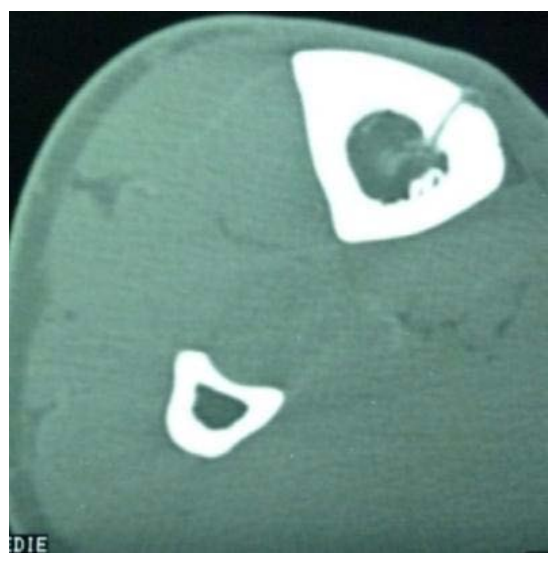

(a)

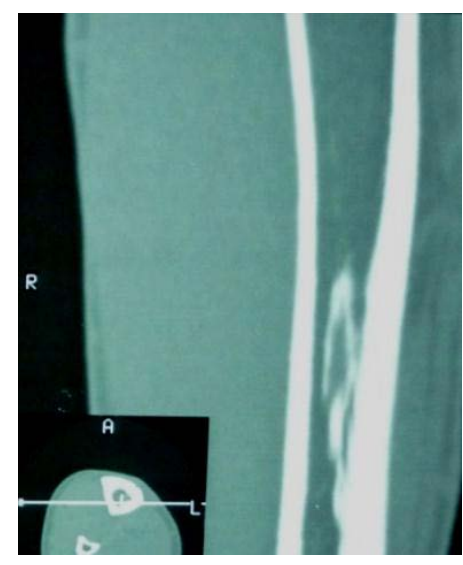

(b)

Figure 2. (a) Axial computed tomographic section, one millimeter thick, showing radiolucent intramedullary gap’s image; (b) Longitudinal computed tomographic section showed spotty internal calcification.

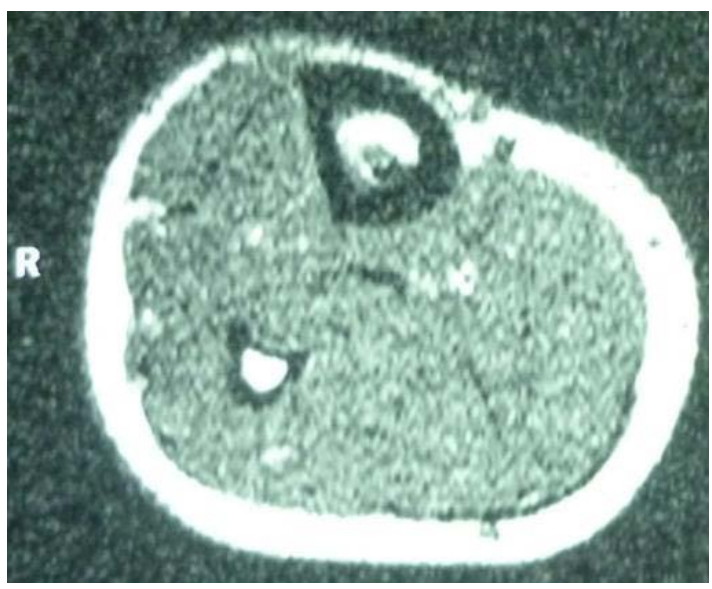

(a)

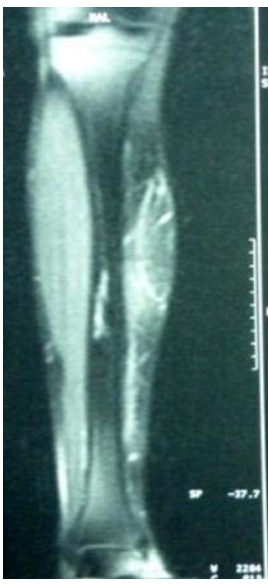

and

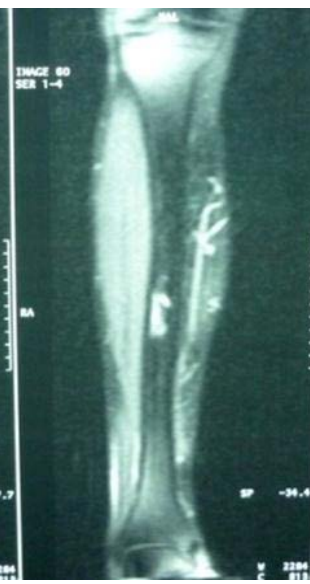

(b)

Figure 3. (a) T1-weighted magnetic resonance image revealing an intramedullary area of hypo signal intensity, with internal, high intense lesion; (b) T2-weighted magnetic resonance image revealing an intramedullary segmental area of high signal intensity.

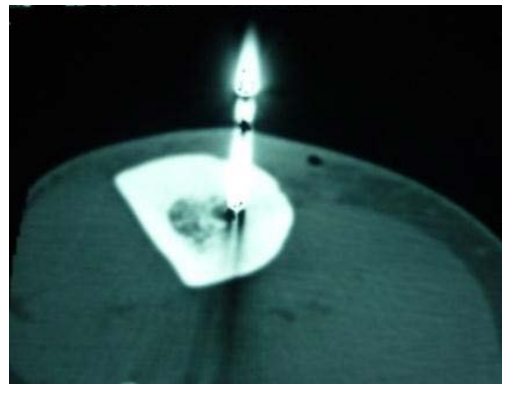

(a)

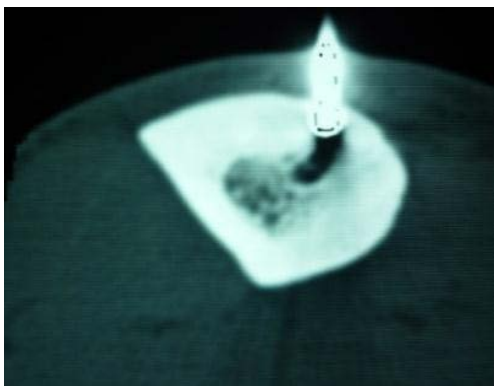

(b)

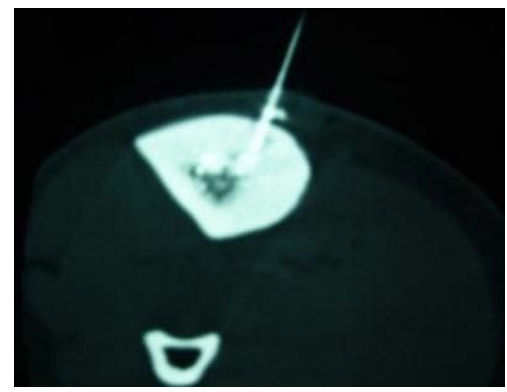

(c)

Figure 4. (a) Catheterization of angioma by a perforated wick guided by a primary trans-cortical pin; (b) The journey was enlarged by a strawberry; (c) Embolisation of intra-medullary angioma.

\section{Discussion}

A haemangioma is an abnormal proliferation of blood vessels that may occur in any vascularised tissue [1]-[4]. Controversial debate is raised as to whether these lesions are neoplasms, hamartomas, or vascular malformations. 
"Haemangiomas” affecting the musculoskeletal system are more accurately termed "vascular malformations." These are present from birth and do not progress spontaneously [1].

Venous haemangiomas are composed of thick-walled vessels containing muscles and they rarely involve the musculoskeletal system. These lesions are generally found in adults and are most frequently located in the deep soft tissues such as the retro peritoneum, mesentery, and muscles of the lower extremities. They often have a slow blood flow, and phleboliths may be present [1]. Haemangioma of the bone is a rare lesion accounting for approximately $1 \%$ of all bone tumors [4]. It may involve the intramedullary canal, the cortical bone, or only the periosteum [5]. These lesions are found most frequently in the vertebrae and skull, most being asymptomatic and discovered only incidentally; they account for $25 \%$ of all skeletal haemangiomas; they occur more frequently in young women and they do not usually require any treatment [5]-[7].

In the long bones, the point of beginning is medullary in $50 \%$ of cases, periosteal in $33 \%$ of cases and cortical in $12 \%$ of cases. The lesion is in the diaphysis or the metaphyso-diaphysis in $80 \%$ of cases and it is isolated metaphysic in $10 \%$ of cases [5]. The possibility of long bone haemangioma is usually not taken into account in the initial diagnosis [8].

Osseous haemangioma which is particularly common in the spine and calvaria affects less frequently long bones such as the tibia, femur, and humerus [1]. Scarcely, the symptoms of osseous haemangioma in the extremities, such as localized swelling, limb hypertrophy, and local pain, are usually nonspecific. Hemorrhage can occur in the setting of trauma, biopsy, or surgery [4].

In our case, there was no osteolysis. The haemangioma is presented as a hair image with peripheral sclerosis. In most cases, haemangioma are presented as osteolysis. The osteolysis was unifocal and diaphyseal [2] [8]. Conventional radiography along with clinical and pathologic data can aid in diagnosing of the wide variety of disease processes that involve the tibial cortex [9].

Bone lysis can have linear and circular components on radiographs, suggestive of a vascular lesion, with linear and circular elements representing vascular channels seen longitudinally and in front respectively. However, these serpentine vascular channels are recognized more easily with CT and MR imaging [5] [8]. Honeycomb, soap bubble, or cystic appearance on radiographs or CT and markedly high-signal intensity on T2-weighted magnetic resonance images (MRIs) have a diagnostic value, but are not decisional for diagnosis [3] [7]. These images were found in our case. Characteristically, these channels have a low signal intensity on T1-weighted images and a very high signal intensity on T2-weighted images because of the slow blood flow. In arteriovenous lesions with a faster blood flow, a low signal intensity may persist with all MR imaging pulse sequences [10] [11].

At the tibia, periosteal or cortical haemangioma occurs most frequently in the anterior tibial diaphysis. These lytic cortical lesions may also show the characteristic multifocal vascular channels or be seen as a larger, nonspecific region of bone destruction. Cortical haemangiomas may predispose the bone to fracture, and periosteal reaction may accompany these lesions [9] [12].

A case of intraosseous arteriovenous malformation (AVM) arising in the diaphysis of the tibia was described by Matsuyama A. Plain radiographs displayed honeycomb lytic lesions in the right tibial shaft, with a slight expansion of the bone and cortical thinning. Curettage of the lesion was carried out, and the histological diagnosis of AVM was made [13].

The surgical treatment is recommended only if the patient is symptomatic or complicated by a pathological fracture [2].

The histopathological diagnosis of a haemangioma is not difficult in a whole sample. The diagnosis from biopsy or tissue curettage is a challenge. It reveals the destructive procedures disrupting thin-walled blood vessels and resulting in histological sections showing non diagnostic empty spaces with scattered bone trabeculae.

Histologically, haemangiomas can be classified as cavernous, capillary, venous or mixed, depending on the type of vascular involvement. Cavernous haemangioma is the most common type in the peripheral bones and accounts for up to $50 \%$ of all cases reported [8]. They are predominant in the medullary and the intracortical portion of the bone and are not yet reported in the diaphysal bone. Pure capillary haemangioma accounts for $10 \%$ of all these types as reported in the literature [11] [13].

Treatment of angiomas is size-dependent. Localized, well-circumscribed lesions are easily treated by cement injection. Larger lesions involving total bones are difficult to treat. Many different treatment options have been reported for haemangiomas of the bone involving the extremities, ranging from simple resection to radiation and even amputation [6] [8] [12]. 
Until recently, predominantly open excisional biopsy was performed, and therefore, surgical treatment was already complete once the definitive diagnosis was revealed by the histological workup [8].

Larger haemangioma in long bones are difficult to treat. If appropriate, the lesions should be excised and packed with bone graft. There are no reports in the literature of limb salvage when haemangioma occurs in nearly the whole long bone [10].

For Cristofaro R [14], selective arterial embolization represents a treatment of choice in aneurysmal bone angioma especially of the spine, sacrum, or pelvis. In these sites embolization replaces surgery which might be hazardous due to intraoperative bleeding. No recurrence was observed in any cases of his study.

There is controversy about the safety of ethanol injections in the treatment of vertebral haemangioma. The study of Bas T [15] shows that intralesional alcohol injections can be considered a safe technique for vertebral haemangiomas. However, a careful technique is required.

Sclerotherapy is the best therapeutic alternative in case of recurrent osseous haemangioma. Under CT guidance the affected bone is punctured by a biopsy needle. The sclerosant is then injected into the haemangioma. Sclerotherapy can be preceded by transcatheter embolization.

CT-guided intraosseous sclerotherapy is a successful therapy. The evolution is often marked by clinical and radiological improvement [16].

In some cases, the management of the haemangioma is made by the association of embolization, sclerotherapy, surgery and radiotherapy.

Cement injection has become a well-accepted treatment option for vertebral pathologies, such as angiomas [16]. The method seems to be well tolerated by the patient and can be done under local anaesthesia. Despite the anatomic proximity of neurologic, vascular structures and the variable high rate of cement extravasations (ranging from $20 \%$ up to $67 \%$ ), the incidence of major complications is very low [1] [16].

\section{Conclusion}

Intramedullary veinous haemangioma of the leg is uncommon with Clinical symptoms mimicking an osteoid osteoma. MR imaging is considered the best mode of evaluating most soft-tissue masses, and haemangioma is no exception. For angiomatous lesions, MR imaging findings are frequently diagnostic. The histological examination confirms the diagnosis. Surgical excision does not prevent recurrence of lesion. Sclerothrapy guided by computed tomographic scan is a good alternative to stabilize intramedullary veinous haemangioma.

\section{Conflict of Interest}

There are no conflicts of interest.

\section{References}

[1] Kenan, S., Abdelwahab, I.F., Klein, M.J. and Lewis, M.M. (1992) Hemangiomas of the Long Tubular Bone. Clinical Orthopaedics and Related Research, No. 280, 256-260. http://dx.doi.org/10.1097/00003086-199207000-00035

[2] Ogose, A., Hotta, T., Morita, T., Takizawa, T., Ohsawa, H. and Harita, Y. (2000) Solitary Osseous Hemangioma outside the Spinal and the Craniofacial Bones. Archives of Orthopaedic and Trauma Surgery, 120, 262-266. http://dx.doi.org/10.1007/s004020050461

[3] Sherman, R.S. and Wilner, D. (1961) The Roentgen Diagnosis of Hemangioma of Bone. The American Journal of Roentgenology, Radium Therapy, and Nuclear Medicine, 86, 1146-1159.

[4] Resnik, D., Kyriakos, M. and Greenway, G.D. (2002) Tumours and Tumour-Like Lesions of Bone. In: Resnik, D, Ed., Diagnosis of Bone and Joint Disorders, 4th Edition, Saunders, Philadelphia, 3979-3985.

[5] Yamamoto, T., Kurosaka, M. and Mizuno, K. (2000) Juxta-Articular Hemangioma of Long Bone. Skeletal Radiology, 29, 535-537. http://dx.doi.org/10.1007/s002560000256

[6] Thomas, A.M.C., Mulligan, P.J. and Jones, E.L. (1990) Benign Haemangioma of Bone in a Middle Phalanx. The Journal of Hand Surgery, 15, 484-486. http://dx.doi.org/10.1016/0266-7681(90)90097-N

[7] Wenger, D.E. and Wold, L.E. (2000) Benign Vascular Lesions of Bone: Radiologic and Pathologic Features. Skeletal Radiology, 29, 63-74. http://dx.doi.org/10.1007/s002560050012

[8] Kaleem, Z., Kyriakos, M. and Totty, W.G. (2000) Solitary Skeletal Hemangioma of the Extremities. Skeletal Radiology, 29, 502-513. http://dx.doi.org/10.1007/s002560000251 
[9] Levine, S.M., Lambiase, R.E. and Petchprapa, C.N. (2003) Cortical Lesions of the Tibia: Characteristic Appearances at Conventional Radiography. RadioGraphics, 23, 157-177. http://dx.doi.org/10.1148/rg.231015088

[10] Yu, L., Cai, L., Yu, G.R., Zeng, Z.H. and Tao, S.X. (2009) Solitary Giant Hemangioma of the Humerus. Orthopedics, 32, 925. http://dx.doi.org/10.3928/01477447-20091020-24

[11] Chawla, A., Singrakhia, M., Maheshwari, M., Modi, N. and Parmar, H. (2006) Intraosseous Haemangioma of the Proximal Femur: Imaging Findings. British Journal of Radiology, 79, 64-66. http://dx.doi.org/10.1259/bjr/53131368

[12] Weiner, D. and Leeson, M.C. (1990) Hemangioma of the Tibia. Orthopedics, 13, 1397-1400.

[13] Matsuyama, A., Aoki, T., Hisaoka, M., Yokoyama, K. and Hashimoto, H. (2008) A Case of Intraosseous Arteriovenous Malformation with Unusual Radiological Presentation of Low Blood Flow. Pathology—Research and Practice, 204, 423-426. http://dx.doi.org/10.1016/j.prp.2008.01.006

[14] Cristofaro, R D., Biagini, R., Boriani, S., Ricci, S., Ruggieri, P. and Rossi, G. (1992) Selective Arterial Embolization in the Treatment of Aneurysmal Bone Cyst and Angioma of Bone. Skeletal Radiology, 21, 523-527. http://dx.doi.org/10.1007/BF00195235

[15] Bas, T., Aparisi, F. and Bas, J.L. (2001) Efficacy and Safety of Ethanol Injections in 18 Cases of Vertebral Hemangioma. Spine, 26, 1577-1582. http://dx.doi.org/10.1097/00007632-200107150-00015

[16] Cohen, J.E., Lylyk, P., Ceratto, R., Kaplan, L., Umansky, F. and Gomori, J.M. (2004) Percutaneous Vertebroplasty: Technique and Results in 192 Procedures. Neurological Research, 26, 41-49. http://dx.doi.org/10.1179/016164104773026516 